\title{
Ocular Complications in Contact Lens Wear and the Risk Factors: A retrospective analysis
}

\author{
Elly Liyana Zainodin ${ }^{1}$, Nur Aresya Ahmad Najmee 1, Farah Najwa binti Hamzah ${ }^{1}$, Noor Haziq Saliman 2,3 \\ ${ }^{1}$ Centre of Optometry, Faculty of Health Sciences, Universiti Teknologi MARA Cawangan Selangor, Kampus Puncak Alam, 42300 \\ Selangor, Malaysia. \\ 2 Institute of Inflammation and Ageing, University of Birmingham, United Kingdom. \\ ${ }^{3}$ National Institute for Health Research-Surgical Reconstruction and Microbiology Research Centre (NIHR-SRMRC), University \\ Hospitals Birmingham NHS Foundation Trust, Birmingham, United Kingdom.
}

ellyzainodin@gmail.com, aresyanajmee@uitm.edu.my, najwahamzahh97@gmail.com, N.H.BinSaliman@bham.ac.uk Tel Author1: 0149678687

\begin{abstract}
Contact lens-related complications might range from harmless irritation to sight-threatening reactions. The key to prevention other than adhering to good hygiene is preliminary eye examinations and timely aftercare assessments. A retrospective analysis of 33 patient clinical records at the university clinic was performed to determine the ocular complications among soft contact lens wearers and its risk factors. Overall, findings indicate that conjunctival hyperemia was the most prevalent complication. Poor lens fit was associated with limbal injection and contact lens papillary conjunctivitis (CLPC). Sleeping with lenses was the most non-compliant behaviour. Eyecare practitioners need to be alarmed by these risk parameters among the young wearers.
\end{abstract}

Keywords: Contact lens; complications; risk factors; hygiene

eISSN: 2398-4287@ 2021. The Authors. Published for AMER ABRA CE-Bs by e-International Publishing House, Ltd., UK. This is an open access article under the CC BYNC-ND license (http://creativecommons.org/licenses/by-nc-nd/4.0). Peer-review under responsibility of AMER (Association of Malaysian Environment-Behaviour Researchers), ABRA (Association of Behavioural Researchers on Asians/Africans/Arabians) and CE-Bs (Centre for Environment-Behaviour Studies), Faculty of Architecture, Planning \& Surveying, Universiti Teknologi MARA, Malaysia.

DOI: https://doi.org/10.21834/ebpj.v6i17.2881

\subsection{Introduction}

Contact lens is one of the common devices used to correct refractive error. It is also beneficial for therapeutic and cosmetic purposes. It is estimated that 125 million people are wearing contact lenses, with the United States leading at 30\% and Singapore at 9\%, and the numbers continue to increase by $6 \%$ every year (Forister et al., 2009; Teo et al., 2011). In some countries, the prevalence is increasing among young adult groups aged 15 to 25 years, dominated by females at $40 \%$ higher than males (Swanson, 2012). In Malaysia, the prevalence of contact lens wearers was at 28\% of its population in 2015 (Morgan et al., 2015). Improvement in terms of the aesthetic, wide range of lens types and materials were the possible factors that influenced the popularity of contact lens usage among students and young working adults (Bowden \& Harknett, 2006).

Non-compliance has been the main issue related to the various aspects of contact lens complications due to the lack of awareness and personal negligence. Contact lens knowledge and awareness are still poor and alarming in certain countries worldwide (Wu, Carnt, \& Stapleton, 2010; Zhu et al., 2018). In Saudi Arabia, approximately 50\% of the wearers were reported to have poor knowledge of the contraindications and possible complications in response to contact lens wear (Alobaidan et al., 2018).

eISSN: 2398-42870 2021. The Authors. Published for AMER ABRA cE-Bs by e-International Publishing House, Ltd., UK. This is an open access article under the CC BYNC-ND license (http://creativecommons.org/licenses/by-nc-nd/4.0/). Peer-review under responsibility of AMER (Association of Malaysian Environment-Behaviour Researchers), ABRA (Association of Behavioural Researchers on Asians/Africans/Arabians) and CE-Bs (Centre for Environment-Behaviour Studies), Faculty of Architecture, Planning \& Surveying, Universiti Teknologi MARA, Malaysia.

DOI: https://doi.org/10.21834/ebpj.v6i17.2881 
In a study among university students, $52 \%$ of the contact lens wearers exhibited some form of non-compliant, especially in lens cleaning and disinfection (Zainodin \& Abdul Hadi, 2020). Similar findings were also reported among college students and healthcare workers at the university hospital where the knowledge and practice of contact lens use and care were below par (de Oliveira, TemporiniNastari, Ruiz Alves, \& Kara-José, 2003).

\subsection{Literature review}

Contact lens wear is known to cause ocular complications due to its direct interaction with the ocular surfaces. The complications can be non-inflammatory or inflammatory, where the latter potentially lead to infections such as microbial keratitis. Other adverse events such as allergic reaction, dry eyes or discomfort have also been reported in the literature (Alipour, Khaheshi, Soleimanzadeh, Heidarzadeh, \& Heydarzadeh, 2017). Despite the indefinite factors to these adverse events, poor care regime, extended-wear, lack of routine eye assessments and poor hand hygiene has shown to be the main contributing factors to the lens-induced complications (Bhandari \& Rou, 2012; Ismail, Rampal, Rahman, \& Ariffin, 2012; Tajunisah, Reddy SC, 2008).

The eye assessments schedule is essential to achieve optimum fitting via a comprehensive contact lens fitting procedure. Poor lens fitting increases corneal staining incidence, allowing bacteria and microorganisms to penetrate the cornea, which eventually causes infection (Young \& Coleman, 2001). It may also increase the symptoms of dryness from the low level of tear exchange in tight lens fitting cases (Carnt, Keay, Naduvilath, Holden, \& Willcox, 2007). Contact lens-related complications and adverse events may also arise from improper lens prescription by unauthorized sellers. Steinemann, Pinninti, Szczotka, Eiferman, \& Price (2003) has revealed the concern on the over-the-counter decorative contact lens by the unauthorized providers with lack of legit information regarding the appropriate lens use and care. This trend where people can acquire contact lenses through the internet without a comprehensive eye examination and correct prescription is worrying as it can contribute to the increment of the prevalence of lens-induced inflammation and infection.

Ocular complications associated with soft contact lens wear vary from mild to severe but are preventable through precise lens fitting, early recognition of complications and guidance on proper hygiene behaviour. The obliviousness of such risk factors among lens wearers, especially the younger generations, increased the probability of severe lens-related complications that can compromise their vision. Investigating the lens-related ocular complications and their enabling factors within young populations is vital, where findings could help practitioners plan the necessary interventions in reducing the risk of non-compliance. This study aimed to explore the ocular surface complications among soft contact lens wearers and its risk factors among the university student population.

\subsection{Methodology}

\subsection{Procedures}

This study involved a retrospective analysis of clinical records of patients attending the university optometry teaching clinic between March 2019 and March 2020. Only patients who wore contact lenses for at least six months prior and designated as regular lens wearers based on the frequency of at least four times per week included in this study. Those who wore contact lenses for a therapeutic reason or have had ocular surface pathologies were excluded from the study. The data on patients' contact lens fitting assessment, and the contact lens-related complications were retrieved from the patient record file based on the aftercare assessments performed during contact lens clinics. The presence of complications extracted was those commonly associated with soft contact lens wear. Data of five complications recorded were bulbar conjunctival hyperemia, limbal injection, contact lens papillary conjunctivitis (CLPC), corneal staining, and corneal neovascularization graded based on the Efron Grading Scale. The scale provides a standard clinical reference for describing the severity of contact lens complications in five stages of increasing severity from 0 to 4 . Patients were noted to have complications if they had an Efron rating of 1 or greater. Information on patient demographics, lens type, location of purchase, wearing schedule, and habits were collected through an online questionnaire adapted from a previous study done by Zhu et al., (2018). Ethics approval was obtained from the UiTM Research Ethics Committee (REC/728/19).

\subsection{Data analysis}

Statistical analyses were performed using SPSS software (ver. 26.0 for Windows; SPSS Inc., Armonk, NY USA) with the significant value set at $p<0.05$. A descriptive analysis was carried out to describe the socio-demographic characteristic, contact lens profile, wearing habits and contact lens-related complications. Analysis of the categorical data was performed using Chi-square to determine whether risk behaviours for contact lens-related complications differ by purchase locations. Fisher Exact Test was used to determine the association between the lens-related complications with wearers' most non-compliant habit and the contact lens fit.

\subsection{Findings}

\subsection{Demographic data}

The total participants in this study were 33 university students, who were predominantly females (93.9\%), aged between 19 to 26 years. All participants were soft contact lens wearers whom the majority were in the bachelor degree programmes (93.9\%). Most of the participants were students from the Faculty of Business and Management $(51.5 \%)$, while the rests were from the Faculty of Health Sciences (30.3\%), Faculty of Education (15.2\%) and Faculty of Accountancy (3\%). 


\subsection{Characteristics of Contact Lens Wearers}

The characteristics of contact lens wearers were evaluated. The majority of the students wore monthly disposable contact lenses (78.8\%), followed by daily disposable (18.2\%) and bi-weekly lenses (3\%). The daily wearing time for most of the students was 6 to 12 hours (78.8\%), with more than half wearing contact lenses for more than two years $(54.5 \%)$. Most of the students purchased their contact lenses from optical shops (54.5\%) and the internet (33.3\%). Only a minority acquired their contact lenses from optometry practice (12.1\%). Comfort $(97 \%)$ and price $(81.8 \%)$ were the two preeminent factors considered for lens selection.

With regards to the wearing and lens care behaviour, most wearers adhered to most aspects of hygiene. In this study, a majority of the lens wearers washed their hands before wearing contact lenses $(61 \%)$, and cleaned their contact lens $(77.8 \%)$ and changed the solution in the lens case (77.8\%) before storage. The habit of swimming with contact lenses was also uncommon among the lens wearers as the majority reportedly had never swim with their lenses (83.9\%). Additionally, all wearers reported they had never cleaned their lenses using tap water. Almost fifty per cent of the wearers sleep with their lenses, making this the wearer's most non-compliant behaviour. Table 1 shows the contact lens wearers' risk behaviour stratified by the location of contact lens purchase. Based on the Chi-Square analysis, there were no significant differences in all behaviours known previously to be the risk factors for contact lens-related complications with the purchase location. Although insignificant, more wearers who did not purchase from optometry practice were non-compliant, particularly in washing hands prior to lens handling, lens cleaning prior storage, and changing lens case solutions.

Table 1. Risk behaviours by contact lens purchase source

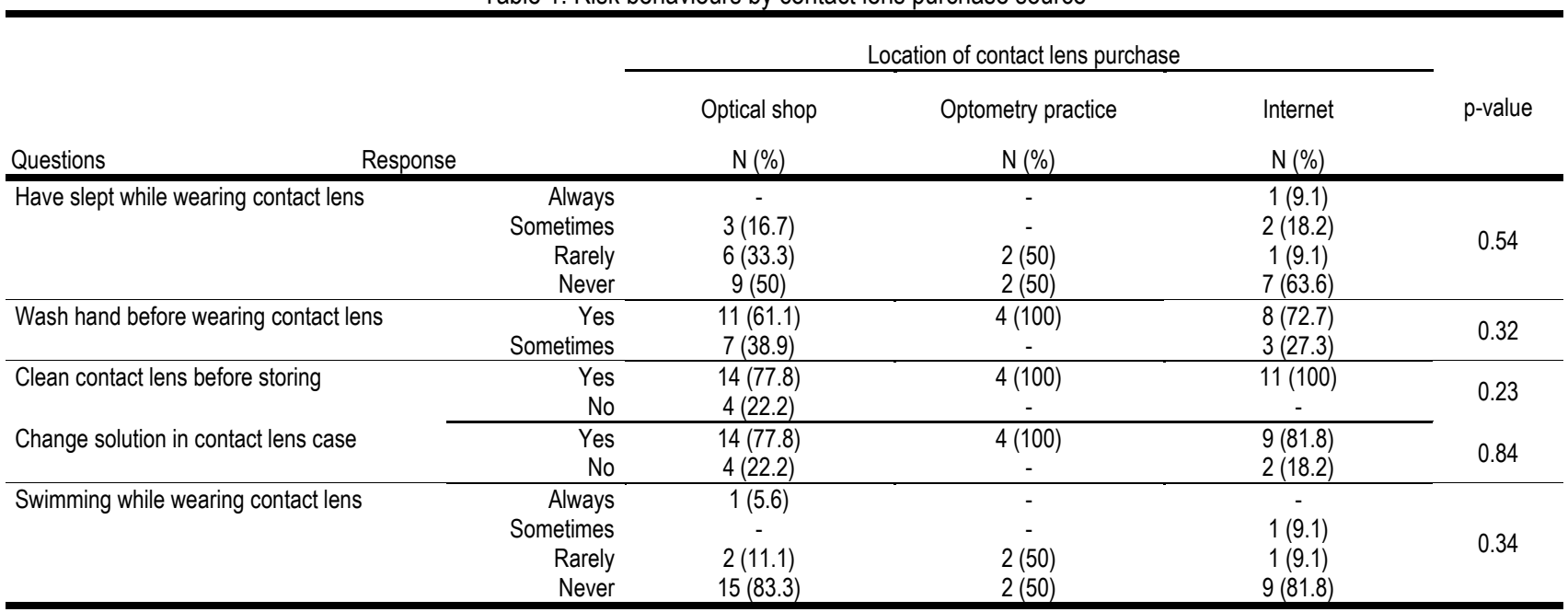

\subsection{Ocular complications related to contact lens use}

Retrospective analysis of the participants' anterior ocular health from their patient record file at UiTM Vision Care Clinic showed the presence of five types of contact lens-related complications that include conjunctival hyperemia, limbal injection, CLPC, corneal staining, and corneal neovascularization. Since the presence of complications was similar in both eyes, only data of right eye was analyzed. Of 33 contact lens wearers, almost half $(48.5 \%)$ had four lens-related complications. The frequency of specific ocular complications is shown in Figure 1. Conjunctival hyperemia was observed as the most prevalent complication (90.9\%), followed by CLPC (84.8\%), corneal staining (81.8\%), and limbal injection (69.7\%). Grading of the complications revealed that most of the participants only had mild (Grade 1 to 2) complications. A moderate or Grade 3 complication was only seen for corneal staining, whereas none had severe complications of Grade 4.

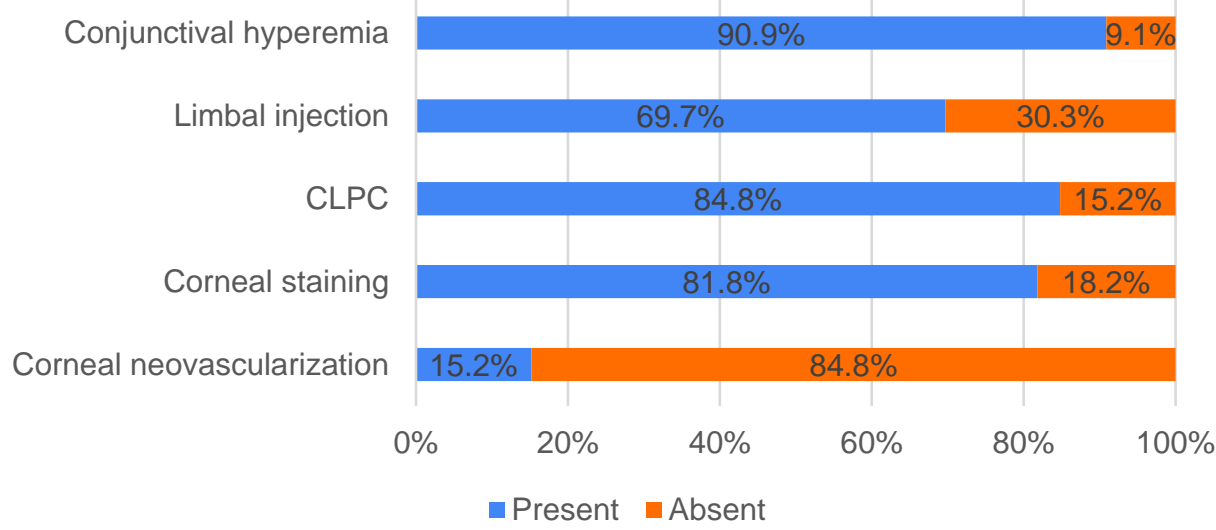

Fig. 1. The frequency of eye complications due to soft contact lens usage 
4.4 Factors associated with ocular complications due to contact use

A Fisher's Exact Test was performed between the ocular complications and the most non-compliant habit exhibited by the lens wearers, sleeping with lenses. There was not a statistically significant association between sleeping with contact lenses and the presence of each ocular complication $(p>0.05)$. With regards to the contact lens fit, a significant association was observed between contact lens fit with limbal injection $(p=0.05)$ and CLPC $(p=0.04)$. Contact lens wearers who wore tight or loose lens fit (not optimum) were more likely to experience these two ocular conditions than those who had an optimum fitting (Figure 2).

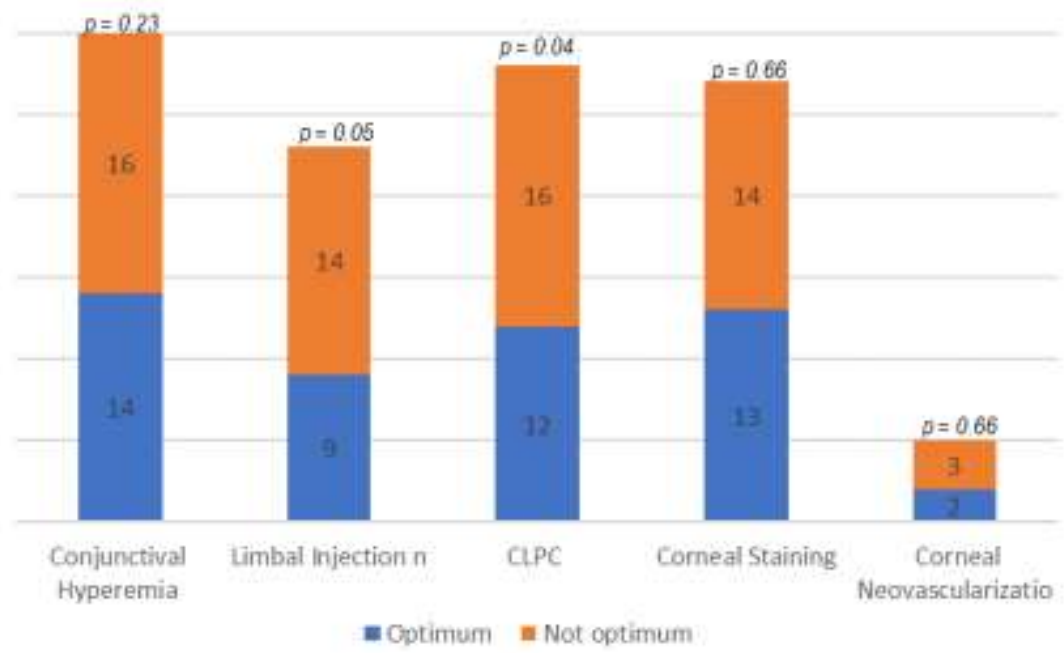

Fig. 2. Ocular complications stratified by type of lens fitting

\subsection{Discussion}

In this study, comfort followed by price were the main factors that lens wearers considered when buying their contact lenses. The result of comfort as an essential factor in choosing contact lenses was consistent with Tajunisah \& Reddy SC, (2008). Lens discomfort has been previously reported as the main reason for contact lens dropout, thus it is not surprising that comfort has become an utmost priority among the wearers (Pucker \& Tichenor, 2020). The lens price was considered the second most important after comfort by the lens wearers in this study, probably due to financial circumstances as they were all students. However, in this study, the wearers' financial standing not acquired, and therefore the relationship of such was not examined.

The most common complication in our analysis among soft contact lens wearers was conjunctival hyperemia (90.9\%), followed by CLPC (84.8\%) and corneal staining (81.8\%). Earlier findings by Forister et al., (2009) cited CLPC and corneal neovascularisation as their most common complications. Our study findings on conjunctival hyperemia are expected as soft lens wearers are prone to suffer from conjunctival hyperemia than rigid lens wearers (McMonnies, C. W., \& Chapman-Davies, 1987) or non-lens wearers (MaldonadoCodina, Morgan, Schnider, \& Efron, 2004), thus providing a higher percentage of the complication. Soft contact lens wearers are inclined to conjunctival hyperemia due to several factors such as corneal hypoxia, lens overwear and poor lens fit (Keir, Woods, \& Sickenberger, 2010).

A significant increase in hyperemia was noted previously with over 6 hours of lens wear (Bruce, 1994) and loose lens fitting (Young \& Coleman, 2001). In this study, the majority reportedly wore their lenses for up to 12 hours, and lens fitting evaluation showed that nearly half of them did not wear properly fitted contact lenses, thus likely contributing to high rates of conjunctival hyperemia.

Sleeping with contact lenses was a frequently reported behaviour that could increase the risk of ocular complications. While $45.5 \%$ of lens wearers in this study reported sleeping while wearing the contact lenses, the findings did not show any significant association with any ocular complications. A possible explanation for this is that although contact lens wearers in this study mostly slept with lenses, such behaviour was not always performed, hence the mild complications. Although this risky soft contact lens wearing behaviour does not imply a causal relationship with any complications, the relationship was evident from previous studies with microbial keratitis and corneal inflammatory events (Chalmers et al., 2011), particularly among the at-risk age group of 15 to 25 -year-old as in this study (Wagner et al., 2014). Sleeping with lenses induced corneal hypoxia and changes to ocular surface biochemistry, making further interaction with contact lenses weakens the cornea's defence mechanism, increases the bacterial adhesion to the ocular surface, and allows progression to microbial keratitis (Fleiszig \& Evans, 2010).

The contact lens fit was found significantly associated with limbal injection and CLPC. Contact lens wearers who wore tight or loose lens fit were prone to acquire these two ocular conditions than those who had an optimum fitting. Our findings are in agreement with Young \& Coleman (2001), where loose lens fit showed a greater amount of limbal injection than those wearing optimum fitting lenses. On the other hand, Sorbara, Maram, Simpson, \& Hutchings, (2018) suggested that wearing tight lens fitting results in a vascular response associated with dryness symptoms. The influence of lens fit on CLPC was previously explained. Compared to aspheric lenses, the nonaspheric lens may produce more edge-lift over the cornea's periphery and limbal, creating a mechanical insult that causes CLPC (Tagliaferri, Love, \& Szczotka-Flynn, 2014). Although the present findings suggest a link between poor lens fitting and CLPC, the theory 
could not be confirmed as no information on the lens design used by the wearers and other causative factors including lens material, modality and care solutions were also not considered.

In optometry practice, a preliminary eye examination, especially the contact lens fitting assessment, helps determine the most suitable contact lens for a patient. However, many people bypass this procedure, buying lenses without comprehensive eye examinations (Zainodin \& Abdul Hadi, 2020). Most lens wearers in this study reflected this behaviour as they mainly purchased their lenses over the counter from the optical shop and the internet. As a result, almost half of them have not worn a suitable contact lens. Additionally, this cohort prone to practising unhygienic behaviours mainly due to not received proper guidance on lens wear and care.

The limitations of the present study are that this is a retrospective analysis where complications were seen only for mild to moderate cases of daily wear, thus not suitable to extrapolate to those using extended wear lenses. The habits of contact lens wear were also solely dependent on a self-reported questionnaire which may introduce bias to the actual behaviour compared to a researcher's observation. Additionally, this study has a small sample size to represent the young adult population, hence again, not suitable to impose the same observations on lens wearers of other age groups. Results are therefore less generalizable. Future research should identify the lens materials and incorporate practitioner's observation in assessing hygiene behaviour on larger samples with age groups diversity.

\subsection{Conclusion \& Recommendations}

Overall, the present study suggests ocular complications, minor to moderate, can occur with daily wear of soft contact lenses. Almost half of soft contact lens wearers had at least four common lens-induced complications. Although these complications were mild and considered non-sight-threatening, they were asymptomatic. It thus accentuates the importance of preliminary eye examinations and aftercare visits with eye care practitioners for regular monitoring. Due to the popular demand for contact lens wear among youth, it is expected that the complications would be more prevalent in this age group. Therefore, attention to the risk factors during patient education may help strengthen the prevention strategies.

\section{Acknowledgements}

We want to thank UiTM Vision Care Clinic for assistance with the patient records.

\section{Paper Contribution to Related Field of Study}

This paper contributes to the field of optometry in Malaysia by providing some perspectives to eye care practitioners on the common ocular surface complications induced by soft contact lens wear and its risk factors. Information emphasizes the current trends and practices that can be used as part of patient consultations.

\section{References}

Alipour, F., Khaheshi, S., Soleimanzadeh, M., Heidarzadeh, S., \& Heydarzadeh, S. (2017). Contact Lens-related Complications: A Review. Journal of Ophthalmic \& Vision Research, 12(2), 193-204. https://doi.org/10.4103/jovr.jovr_159_16

Alobaidan, O. S., Alkhalifah, M. K., AlSayegh, A. A., Alhumaid, F. A., Ashammery, A. S., Alghamdi, K., ... AlRashid, W. (2018). Knowledge and practice regarding contact lens among Saudi urban contact lens users. Saudi Journal of Ophthalmology: Official Journal of the Saudi Ophthalmological Society, 32(2), 93-96. https://doi.org/10.1016/j.sjopt.2017.09.008

Bhandari, M., \& Rou, H. P. (2012). Habits of contact lens wearers toward lens care in Malaysia. Medical Journal of Malaysia, 67(3), 274-277.

Bowden, T., \& Harknett, A. (2006). What the patient wore, and why \&\#x2026; Contact Lens and Anterior Eye, 29(1), 5-15. https://doi.org/10.1016/j.clae.2005.12.002

Bruce, A. (1994). Soft contact lens design and bulbar conjunctival hyperemia. American Academy of Optometry. Retrieved from https://www.aaopt.org/detail/knowledgebase-article/soft-contact-lens-design-and-bulbar-conjunctival-hyperemia

Carnt, N. A., Keay, L., Naduvilath, T., Holden, B., \& Willcox, M. (2007). Risk Factors Associated With Corneal Inflammation in Soft Contact Lens Daily Wear. Investigative Ophthalmology \& Visual Science, 48(13), 4326

Chalmers, R. L., Wagner, H., Mitchell, G. L., Lam, D. Y., Kinoshita, B. T., Jansen, M. E., .. McMahon, T. T. (2011). Age and Other Risk Factors for Corneal Infiltrative and Inflammatory Events in Young Soft Contact Lens Wearers from the Contact Lens Assessment in Youth (CLAY) Study. Investigative Ophthalmology \& Visual Science, 52(9), 6690-6696. https://doi.org/10.1167/iovs.10-7018

de Oliveira, P. R., Temporini-Nastari, E. R., Ruiz Alves, M., \& Kara-José, N. (2003). Self-Evaluation of Contact Lens Wearing and Care by College Students and Health Care Workers. Eye \& Contact Lens, Retrieved https://journals.Iww.com/claojournal/Fulltext/2003/07000/Self_Evaluation_of_Contact_Lens_Wearing_and_Care.4.aspx

Fleiszig, S. M. J., \& Evans, D. J. (2010). Pathogenesis of contact lens-associated microbial keratitis. Optometry and Vision Science : Official Publication of the American Academy of Optometry, 87(4), 225-232. https://doi.org/10.1097/OPX.0b013e3181d408ee

Forister, J. F. Y., Forister, E. F., Yeung, K. K., Ye, P., Chung, M. Y., Tsui, A., \& Weissman, B. A. (2009). Prevalence of Contact Lens-Related Complications: UCLA Contact Lens Study. Eye \& $\quad$ Contact $\quad$ Lens, $\quad$ 35(4). 
https://journals.Iww.com/claojournal/Fulltext/2009/07000/Prevalence_of_Contact_Lens_Related_Complications_.4.aspx

Ismail, L., Rampal, L., Rahman, A., \& Ariffin, A. (2012). Non-Compliance to Lens Care Procedures in Patients with Contact Lens Related Microbial Keratitis. 5, 14-20.

Keir, N., Woods, J., \& Sickenberger, W. (2010). The conjunctival response to soft contact lens wear: A practical guide. 11.

Maldonado-Codina, C., Morgan, P. B., Schnider, C. M., \& Efron, N. (2004). Short-Term Physiologic Response in Neophyte Subjects Fitted with Hydrogel and Silicone Hydrogel Contact Lenses. Optometry and Vision Science, 81(12). https://journals.Iww.com/optvissci/Fulltext/2004/12000/Short_Term_Physiologic_Response_in_Neophyte.8.aspx

McMonnies, C. W., \& Chapman-Davies, A. (1987). Assessment of conjunctival hyperemia in contact lens wearers. Part II. American Journal of Optometry and Physiological Optics, 64(4), 251-255. https://doi.org/https://doi.org/10.1097/00006324-198704000-00004

Morgan, P., Woods, C., Tranoudis, I., Helland, M., N, E., Jones, L., ... J, N. (2015). International Contact Lens Prescribing in 2015. Contact Lens Spectrum, 31(January 2016), 24-29. Retrieved from https://www.clspectrum.com/issues/2016/january-2016/international-contact-lens-prescribing-in-2015

Noushad, B., Saoji, Y., Bhakat, P., \& Thomas, J. (2012). Contact lens compliance among a group of young, university-based lens users in South India. Australasian Medical Journal, 5, 168-174. https://doi.org/10.4066/AMJ.2012.104

Pucker, A. D., \& Tichenor, A. A. (2020). A Review of Contact Lens Dropout. Clinical Optometry, 12, 85-94. https://doi.org/10.2147/OPTO.S198637

Sorbara, L., Maram, J., Simpson, T., \& Hutchings, N. (2018). Corneal, Conjunctival effects and blood flow changes related to silicone hydrogel lens wear and their correlations with end of day comfort. Contact Lens and Anterior Eye, 41(2), 193-200. https://doi.org/10.1016/j.clae.2017.12.010

Steinemann, T. L., Pinninti, U., Szczotka, L. B., Eiferman, R. A., \& Price, F. W. J. (2003). Ocular Complications Associated with the Use of Cosmetic Contact Lenses from Unlicensed Vendors. Eye \& Contact Lens, Retrieved 29(4). https://journals.Iww.com/claojournal/Fulltext/2003/10000/Ocular_Complications_Associated_with_the_Use_of.2.aspx

Swanson, M. W. (2012). A Cross-Sectional Analysis of U.S. Contact Lens User Demographics. Optometry and Vision Science, 89(6). Retrieved from https://journals.Iww.com/optvissci/Fulltext/2012/06000/A_Cross_Sectional_Analysis_of_U_S_Contact_Lens.5.aspx

Tagliaferri, A., Love, T. E., \& Szczotka-Flynn, L. B. (2014). Risk Factors for Contact Lens-Induced Papillary Conjunctivitis Associated With Silicone Hydrogel Contact Lens Wear. Eye \& Contact Lens, 40(3). Retrieved from https://journals.Iww.com/claojournal/Fulltext/2014/05000/Risk_Factors_for_Contact_Lens_Induced_Papillary.1.aspx

Tajunisah, Reddy SC, P. S. (2008). Knowledge and practice of contact lens wear and care among medical students of University of Malaya. Medical Journal of Malaysia3, 63(3).

Teo, L., Lim, L., Tan, D. T. H., Chan, T.-K., Jap, A., \& Ming, L. H. (2011). A Survey of Contact Lens Complications in Singapore. Eye \& Contact Lens, 37(1). Retrieved from https://journals.Iww.com/claojournal/Fulltext/2011/01000/A_Survey_of_Contact_Lens_Complications_in.5.aspx

Wagner, H., Richdale, K., Mitchell, G. L., Lam, D. Y., Jansen, M. E., Kinoshita, B. T., ... Group, for the C. S. (2014). Age, Behavior, Environment, and Health Factors in the Soft Contact Lens Risk Survey. Optometry and Vision Science, 91(3). Retrieved from https://journals.Iww.com/optvissci/Fulltext/2014/03000/Age,_Behavior,_Environment,_and_Health_Factors_in.3.aspx

Wu, Y., Carnt, N., \& Stapleton, F. (2010). Contact lens user profile, attitudes and level of compliance to lens care. Contact Lens and Anterior Eye, 33(4), 183-188. https://doi.org/10.1016/j.clae.2010.02.002

Young, G., \& Coleman, S. (2001). Poorly fitting soft lenses affect ocular integrity. The CLAO Journal: Official Publication of the Contact Lens Association of Ophthalmologists, 27(2), 68-74.

Zainodin, E. L., \& Abdul Hadi, N. S. (2020). Non-compliance Behavior in Contact Lens Wear and Care among University Students . Environment-Behaviour Proceedings Journal, 5(14 SE-), 11-18. https://doi.org/10.21834/ebpj.v5i14.2192

Zhu, Q., Yang, B., Deng, N., Li, Y., Wang, T., Qi, H., \& Liu, L. (2018). The use of contact lenses among university students in Chengdu: Knowledge and practice of contact lens wearers. Contact Lens and Anterior Eye, 41(2), 229-233. https://doi.org/10.1016/j.clae.2017.12.008 